factors and HIV-1 subtypes among crack users in Goiania, a city in Midwestern Brazil.

Methods A total of 600 crack users were interviewed and blood samples were collected for detection of anti-HIV-1. HIV-RNA was detected in positive samples, the HIV-1 protease (PR) and reverse transcriptase (RT) regions were sequenced; subtypes were assigned by REGA/phylogenetic analysis. HIV-subtypes were assigned by REGA. Univariate and multivariate analysis were carried out to identify predictors of HIV infection. This study was analysed and approved by the Committee on Ethics in Human Research of Hospital das Clínicas, Universidade Federal de Goiás.

Results The median age of participants was 30 years old, and the majority were male, mixed race, single, and unemployed. Of the total, $2.8 \%$ were HIV-positive. Having sex with an HIV carrier, irregular condom use during sexual intercourse, and experience of living on the streets were predictors of HIV positivity $(\mathrm{p}<0.05)$. In 12 out of 17 crack users RNA HIV-1 were amplified and sequenced. Seven isolates were subtype B, one subtype F1 and one subtype C.

Conclusion A high HIV prevalence was observed among crack users in Midwestern Brazil, a region far from the epicentre of HIV epidemiology. The predictors of HIV infection identified are concerning, and necessitate preventive strategies for HIV infection specifically directed toward this population. HIV-1 subtype $\mathrm{C}$ seems to have emerged over the last few years in this population at the centre of the country.

Support: This work was supported by Conselho Nacional de Desenvolvimento Cientifico e Tecnologico - CNPq, and Fundação de Amparo a Pesquisa do Estado de Goiás - FAPEG

\section{P3.204 PREVALENCE AND RISK FACTORS FOR SYPHILIS IN CRACK USERS IN GOIÂNIA CITY, BRAZIL CENTRAL}

'Sheila Araujo Teles, ${ }^{1}$ Rafael Alves Guimarães, 'Divânia Dias da Silva França, ${ }^{2}$ Leandro Nascimento Silva, ${ }^{1}$ Megmar Aparecida Dos Santos Carneiro, ${ }^{1}$ Nativa Helena A Del Rios, ${ }^{1}$ Lyriane Apolinário Araujo, ${ }^{1}$ Marcos André de Matos, ${ }^{1}$ Karlla Antonieta Amorim Caetano. ${ }^{1}$ Universidade Federal de Goiás, Goiânia - GO, Brazil; ${ }^{2}$ Secretaria Municipal de Saúde de Goiânia, Goiânia - GO, Brazil

\subsection{6/sextrans-2017-053264.439}

Introduction Crack users are at high risk for syphilis, due to multiple risk behaviours, such as inconsistent condom use, exchanging sex for money and/or drugs and multiple sexual partners. However, there is a gap in the literature on the epidemiology of this infection in crack users in developing countries, including Brazil. The aim of this study was to investigate the prevalence and risk factors of syphilis in crack users in Goiânia, Brazil Central.

Methods A cross-sectional study was conducted with 600 users of a reference unit for chemical dependency treatment of Goiás, between 2012 to 2013. All participants were interviewed regarding socio-demographic characteristics and risk behaviours and blood samples were then collected for the detection of anti-Treponema pallidum antibodies using enzymelinked immunosorbent assay (ELISA). Positive samples were tested by the Venereal Disease Research Laboratory (VDRL). Active syphilis was considered when there was a positive ELISA result and VDRL titers $\geq 1: 8$. Poisson regression analysis was used to analyse the factors associated with active syphilis.
Results Of the total participants, $13.8 \%(95.0 \%$ CI: $11.3 \%$ to $16.8 \%$ ) were positive in ELISA. The prevalence of active syphilis was $4.5 \%$ (95.0\% CI: $3.1 \%$ to $6.5 \%)$. The prevalence of exposure to Treponema pallidum and active syphilis was statistically higher in women than in men $(\mathrm{p}<0.001)$. In multivariable model, female sex (adjusted prevalence ratio [APR]: 3.73; $\mathrm{p}<0.001$ ), schooling (APR: 0.88; $\mathrm{p}=0.030$ ), exchange sex for money and/or drugs (APR: 3.22; $\mathrm{p}=0.040$ ) and a history of genital ulcers in previous six months (APR: 4.12; $\mathrm{p}=0.010$ ) were associated with active syphilis.

Conclusion The results of this research show high prevalence of syphilis in crack users in Goiânia city. Comprehensive care for this population should be offered, including strategies such as health education, condom provision, and regular infection testing.

Support: Fundação de Amparo a Pesquisa do Estado de Goiás - FAPEG; Conselho Nacional de Desenvolvimento Científico e Tecnológico - CNPq

\section{P3.205 AUGMENTED PARTNER RISK FEATURES BETWEEN TEENAGERS USING ALCOHOL IN THE MOMENT}

${ }^{1}$ Shkurti Enkelejda, ${ }^{2}$ Shtiza Diamant. ${ }^{1}$ University of Medicine, Tirana - Albania; ${ }^{2}$ University Hospital Centre Mother Theresa, Tirana - Albania

\subsection{6/sextrans-2017-053264.440}

Introduction Alcohol is an acknowledged risk factor for sexually transmitted illnesses achievement, but the device is unclear. Potentially, teenagers using alcohol in the 2 hours previous to sex (in-the-instant use) have riskier sexual partners. Methods We applied multivariable logistic regression to observe the connexion among in-the-instant alcohol use and partner risk features accounted for the most current sex between principally 16 - to 17-year-old teenagers initially engaged from a representative trial of Tirana public elementary schools. We generated 3 combined partner risk outlines: partner awareness risk (informal and unpredicted), partner situation risk (age discordance and met in public), and generally risk using all measures excluding partner alcohol use.

Results Adolescents who reported any in-the-instant alcohol use were more expected to nondrinking youngsters to state casual (adjusted odds ratio [AOR], 3.1; 95\% confidence interval [95\% CI], 1.9-5.0), unpredicted (AOR, 1.5; 95\% CI, 1.22.7), age dissonant (AOR,2.8; 95\% CI, 1.9-4.5), or met in public partners (AOR, 1.4; 95\% CI, 1.0 to 2.1). For each compound evaluation, the number of partner hazard features accounted augmented linearly with the percentage of teenagers drinking in the moment (Cochran-Armitage trend, $\mathrm{p}<0.0001$ ). In relation to zero characteristics, in-the-moment alcohol use was connected with increased odds of reporting 1 (AOR, 2.5; 95\% CI, 1.5-4.8), 2 (AOR, 4.3; 95\% CI, 2.8, 7.7).

Conclusion The results develop the association among in-themoment alcohol utilisation and partner hazard accounted in previous surveys to include adolescents' universal sexual knowledge and supplementary partner features counting the extremely allied compound features

Support: The Melon Institute and Metabolism Corp are funded by the University of Oxbridge, UK 